\title{
Working from home during COVID-19 pandemic: lessons learned and issues
}

\author{
Ettore BOLISANI \\ University of Padova, Vicenza, Italy \\ ettore.bolisani@unipd.it \\ Enrico SCARSO \\ University of Padova, Vicenza, Italy \\ Christine IPSEN \\ Technical University of Denmark, Lyngby, Denmark \\ Kathrin KIRCHNER \\ Technical University of Denmark, Lyngby, Denmark \\ John Paulin HANSEN \\ Technical University of Denmark, Lyngby, Denmark
}

\begin{abstract}
During the COVID pandemic, many companies, schools, and public organizations all around the world asked their employees to work from home i.e. to adopt what are called "smart working" modalities. This has and will presumably have a serious impact on both employees and employers, which still needs to be clarified and investigated: indeed, if smart working becomes a common working modality, this may have a significant impact on both organizations and employees. This paper reports the results of an online survey of "smart workers" in Italy during the COVID pandemic, when a great number of employees suddenly moved to working from home with no or little preparation. The study offers interesting indications about the involvement and usefulness perception of smart working by the sampled people and makes it possible to single out different categories of employees based on their attitude towards this modality. Also, it points out the potential impact on socialization among colleagues, and the consequent implications for knowledge sharing and knowledge management. From the collected responses, a fully positive or negative conclusion about working from home was not possible, nor a clear indication about the efficiency and effectiveness of this working modality. The analysis, instead, highlighted the presence of different but numerically similar groups of people, i.e. those who were not satisfied at all with the experience, those who were very satisfied, and those who were "undecided". Furthermore, respondents underlined the importance and the difficulty to maintain working contacts and the intense use of communication systems made for this purpose. Lastly, collected opinions on positive and negative aspects of working from home provided some practical suggestions about how to successfully implement this solution.
\end{abstract}

Keywords: COVID-19, working from home, communication tools, smart working, knowledge workers.

Please cite the article as follows: Bolisani, E., Scarso, E., Ipsen, C., Kirchner, K. and Hansen, J.P (2020), "Working from home during COVID-19 pandemic: lessons learned and issues", Management \& Marketing. Challenges for the Knowledge Society, Vol. 15, No. Special Issue, pp. 458-476, DOI: 10.2478/mmcks-2020-0027. 


\section{Introduction}

During the COVID pandemic, many companies, schools, and public organizations all around the world asked their employees to start working from home or "smart working" (SM) (Brynjolfsson et al., 2020; Gottlieb et al., 2020). This has and will presumably have a serious impact on both employees and employers, which still needs to be clarified and investigated.

Before the COVID crisis, compared to the figures we have today, the number of SM employees was much smaller. In Italy for example - the country which is the main target of this analysis - the number more than doubled with the pandemic compared to the previous year (source: Observatory on Smart Working, Milan Polytechnic, www.osservatori.net) and reached more than one million people in just a few weeks. People for whom, in the past, SM was substantially unknow, had suddenly start adopting this new modality.

It is difficult to predict the future, but if after the pandemic SM remains a usual way of working especially for clerical workers, this will have a significant impact on the way working activities are managed in companies and public organizations. So, the current figures urge to conduct research on the reactions of people to that sudden change in work modality, and on the actual impact on their working and lifestyle.

This paper reports the results of an online survey conducted in many different countries. The focus here is Italy, where almost 1000 responses were collected, which provides a significant basis. The aim of this research was to provide descriptive statistics of the involvement and usefulness perception of SM and, furthermore, to single out different categories of employees based on their attitude towards SM. The ultimate goal is to contribute to the research on SM, especially by analyzing the impact of a sudden move towards this work modality as is happening in these days, and to provide insights into the new challenges for management that these changes can pose.

\section{Literature review}

A first clarification regards the terms that are used in this paper. Working "from home" has often been referred to in different ways. Especially in the past, a popular term often used in this context was "telework", and while there is no universal agreement (Alizadeh, 2013), it is generally described as a way to work "away from the office" by means of electronic connections. Malik et al. (2016) argue that this implies that employees can work not only from home but also from any other places that are not the normal office of the company (for example, a satellite office or an external location).

Smart working can be regarded as an evolution of teleworking, but the adjective "smart" puts an emphasis on the potentially positive effects that this new modality is expected to bring about, for both companies and people: Kim et al. (2017) argue that SM is "telework that individuals perform in a smart and innovative manner" by using devices for distant communication. From this viewpoint, it is highlighted that workers "can positively accommodate the latest information technology environment without being constrained by time and place" (Kang and Kwon, 2016). The adjective "smart" is used to underline that this work modality is also a way for employees to "achieve maximum value" and "be more productive" (Fragouli and Ilia, 2019), to have more "spatial and temporal flexibility" (Fogarty et al., 2011), better working conditions (Kim and Oh, 2015), improve creativity and stimulate change (Kang and Kwong, 2016). In summary, in this study, we prefer to use the term Smart Working (SM) rather than "teleworking", because it not only emphasizes 
"working at distance" and the use of telecommunication technologies, but also the potential effects of this work modality to employees and employers, and the changes it can induce.

Especially, we will focus on these aspects of SM seen as a modality that implies:

a) for employees, mainly working from home

b) for employers, adopting appropriate changes in working procedures and task in case and where this is functional to the effectiveness of the job tasks that are performed

c) for both employees and employers, employing an effective combination of information technologies, computer networks, and mobile devices.

In the rest of the paper, we will preferably refer to SM, although for our purposes the term "working from home" will be used as a synonym. We are especially interested in analyzing the effects of SM on employees and on their "perception of work". This topic is important for research but also for companies, which can learn the potential importance but also the possible problematic implications of the introduction of SM modalities in their organizations, and consequently can focalize the critical points to be considered for a successful SM implementation.

The potential impacts of SM on employees and the factors that can influence its success (for employees and for firms) has already been considered in the literature. Hassan (2016) detected a number of significant factors, namely: nature of the job, clear goals and policies, fitting to immediate needs of employees, and also a capability to respect the personal like of the people. Similarly, Kang and Kwon (2016) analyzed the possible determinants of a positive adoption of SM, and singled out that there are factors at firm level (such as, the organizational environment - e.g.: innovation climate, or style of personnel evaluation - or the characteristics of the information system adopted to support $\mathrm{SM}$ ), and others at the level of the single individual (for example, capability of self-control).

The possible differences in acceptance and perception between employees have also been widely considered. SM efficacy can depend on the specific work organization. For instance, van der Lippe and Lippényi (2020) signal that it can make it difficult to work in team when there are too many employees who work from home. Also, the job position (Park, 2018) and the leadership style of each job and individual (Park and Kim, 2013) can play a role. It is also worth recalling that SM can have an impact on socialization processes and personal work styles (Mallia and Ferris, 2000; Troup and Rose, 2012). Indeed, SM may change the forms of interaction between colleagues, which in turn can modify the processes of knowledge sharing and knowledge management. Effectiveness and quality of work especially (but not only) for knowledge workers (Bélanger and Allport, 2008) - can be consequently affected. The knowledge management literature provides useful insights into this issue. For instance, Dalkir (2008) used the distinction between tacit and explicit knowledge and Nonaka's (1994) model of knowledge conversions to explain that different information technologies can be more or less effective for interpersonal knowledge sharing, in relation to their capability to transfer tacit or explicit contents efficaciously. From another perspective, by adopting Bratianu and Bejinaru's $(2019,2020)$ metaphor of knowledge as energy field, it may be argued that the use of SM can require that employees find a new dynamic balance between the different forms of knowledge (i.e. rational, emotional, and spiritual) which characterize actions and decisions of people.

As mentioned, although the adjective "smart" may suggest that SM can be beneficial to companies and employees, it is also important to check if this modality may, conversely, find implementation obstacles or lead to additional problems in work, work management, 
and also private lives of employees. This point is still debated in the literature. Some studies underline the positive effects of SM in association to the increased flexibility and the potentially better work conditions (Govindaraju and Sward, 2005), others its difficult implementation or negative impacts (Richardson et al., 2006). Possible obstacles to successful implementation can include the required investments in information technologies, but also organizational aspects e.g. difficult coordination and cooperation among workers, fear of loss of control by management, and anxiety related to work in isolation (Sarti and Torre, 2017). The impact on individual life is also a critical issue (Dockery and Bawa, 2014). On the one hand, SM is commonly seen to provide better tradeoff between work and private life: the ability to work, at least in part, from home can be beneficial to employees because it can provide flexibility and can help them to balance work and private or family life. On the other hand, working from home is often associated with longer hours of work, and concerns that SM can lead to greater intrusion of working issues into the personal space of people.

To sum up, the literature highlights some important points. Firstly, the effective and successful use of SM can depend on various factors that can be very different and partially independent from one another, and especially: the kind of job that is performed in the SM modality, the organizational characteristics and working environment, the clarity of goals and policies, the information technologies that a company implements to support SM, and also the single individual's attitude and lifestyle. Secondly, SM impacts on the socialization processes of employees. This, in turn, can affect the effectiveness of knowledge sharing that is required to profitably perform a specific task. Thirdly, while some studies tend to highlight the benefits of SM, others underline that there may be obstacles and negative effects, for companies and individuals, so this point is controversial.

The basic starting point of this study is the observation that all the mentioned studies refer to a "pre-COVID" time: in other words, to situations where working in the office was the normality, and only a small fraction of employees were using SM modalities (generally on a voluntary basis). With the pandemic, the situation is completely different. Many companies, organization, and individuals suddenly needed to adopt SM very quickly, with little experience and expertise. It is possible that, in this forced condition, SM becomes a positive opportunity for change (Langè and Gastaldi, 2020), but this cannot be taken for granted.

Therefore, there is much to learn about the effects of SM and the perceptions of employees in this "forced" conditions, because this can help to explain the possible paths to a successful SM implementation. Also, it can be useful to understand if SM can be a real opportunity for everybody, or if there are specific conditions and potential issues that should be considered at least for specific categories of employees, firms, and jobs.

\section{Research goals and method}

This study presents the Italian part of an international survey aimed to learn from the people's experience when they were working from home during the COVID-19 outbreak. It is an exploratory research based on an online questionnaire administered almost immediately after the lockdown (middle of March 2020). Given the need to discuss the survey's outcomes immediately, a preliminary analysis of the collected data is shown here; more sophisticated elaborations will be performed later. Particularly, we calculated the distribution of the responses, applied a cluster analysis, and a discriminant analysis to 
verify if it was possible to identify the presence of distinct groups of people with similar opinions, behaviors, and so on.

Cluster analysis is a well-known quantitative social research method (Sing 2007). It allows to discover groups (clusters) within a complex body of data. A large number of observations (i.e.: the answers given to the questionnaire by each respondent) concerning a set of variables (so called "base variables", namely the main items of the questionnaire) is reduced into smaller groups so that some significant features within each group is relatively similar while being relatively dissimilar across different groups (Sing 2007; Kaufman and Rousseeuw, 2009; Everitt et al., 2011). This makes it possible to single out the different clusters in a sample, and to discriminate between recurring structural or behavioral patterns. In our case, this makes it possible to discover if there are groups of respondents who gave similar responses, and to characterize these groups based on that. A discriminant analysis can be employed to describe the discovered clusters by profiling them based on other descriptors - in our case, aspects as e.g. age, education level, number of family members etc. of the interviewed. Typically, the average value of both base and descriptor variables are reported in the profile of each cluster (Kaufman and Rousseeuw, 2009).

Our survey was based on a questionnaire of 23 questions (mostly closed) concerning opinions about working from home, use of digital collaboration technology, and some essential demographic data. With the aim of encouraging a large participation, the number of questions was small, and anonymity was guaranteed. The questionnaire consisted of some distinct sections, namely: current work situation, perceived advantages of working from home, perceived disadvantages, demographic data, and perceptions of the overall situation. Further information about the questionnaire, its aim, theoretical background, and validation of the single questions, can be found in Ipsen et al. (2020).

The largest part of the questions required a response based on a 5-point Likert scale, where respondents were asked to indicate how much they agreed to a statement. To process the cluster analysis, the original scales were converted to normalized numeric values (from 1 to 5). Similar conversions were made for items as age, education level, number of contacts, etc. A few questions asked a specific numeric value (for example, number of weekly days of SM) or a yes/no choice. The questionnaire also had four open questions (whose results are not analyzed here for reason of space).

To capture the early feelings of people working from home, the survey was promoted through the personal network of the authors (which include Industry Associations, Student Associations, Academics Associations, Individual companies, LinkedIn contacts and so on) and by using a "snowball effect" (i.e. respondents were also asked to invite their friends to respond to the survey). Consequently, there is a bias because responses mainly came from "knowledge workers", i.e. people whose job has intellectual or clerical contents. In any case, the adopted method made it possible to collect a relevant number of responses which provide an interesting picture of the investigated matter.

The questionnaire was used in all the other countries involved in the survey. It was originally formulated in English (Ipsen et al., 2020) and then translated in Italian. The large majority responded to the Italian version of the questionnaire, only a few responded to the English version.

The English survey started on 20th March 2020. Four days later, a first response by an Italian employee was received. The Italian version was issued on 5th April 2020. The last response (included in the statistics presented here) refers to the 13th May 2020. A few 
responses were submitted after this date and were not considered in this analysis. In total, 931 responses of Italian employees were elaborated. The demographic characteristics of the sample are shown in table 1.

Table 1. Sample demographics

\begin{tabular}{|l|l|l|}
\hline Variable & Category & N. of respondents \\
\hline Age (years) & $18-40$ & 127 \\
& $40-50$ & 240 \\
& $50-60$ & 309 \\
& $>60$ & 205 \\
& n.a. & 60 \\
\hline Gender & Female & 376 \\
& Male & 542 \\
& Other & 12 \\
& n.a. & 1 \\
\hline Level of education & Doctorate & 37 \\
& Master & 459 \\
& Bachelor & 122 \\
& High School & 251 \\
& Lower & 51 \\
\hline Type of work & Student & 25 \\
& Administrative & 205 \\
& Research & 65 \\
& Systems or plan development & 88 \\
& Manager & 157 \\
& Communication & 20 \\
& Teaching & 50 \\
& Commercial business & 103 \\
& Systems monitoring & 76 \\
& Manufacturing & 131 \\
& Creative production & 3 \\
& Other & 240 \\
\hline \multirow{5}{*}{} & & (a) \\
\hline
\end{tabular}

Source: Authors' own research results

The sample is made of people of relatively mature age. The biggest age group is between 50 and 60, while the smallest is that between 18 and 40. Respondents are well balanced as regards gender, but not in terms of level of education: the largest (around 50\%) is composed by individuals with a university master's degree. Different kinds of job are included (note that multiple answers were possible), and particularly: administrative positions (22.0\%), management positions (16.9\%), manufacturing jobs (14.1\%). A significant share of respondents (25.8\%) indicated "other", although the complete description of their job (as was requested in this case) just recalled one of the other types. $21 \%$ of respondents affirmed to live alone, while $37 \%$ with children under 15 years.

Table 1 confirms that this is not a random sample of the Italian labor force, because it includes mostly highly educated professionals and managers, and a higher share of women. The response group is selective and especially indicative of knowledge workers, as previously mentioned. Indeed, this category of employees is that for which SM is more likely, which makes the results of this analysis significant. Nonetheless, this bias must be considered in the interpretation of findings. 


\section{Findings}

In this paper we present some preliminary elaborations that are however particularly useful to show some interesting points of the investigated issues. In particular, we excluded from the analysis the answers to questions related to general impacts of the COVID 19 situation (for example, whether respondents thought that SM reduces the risk to spread the virus, or that it can limit the possibility of physical exercise) because the study specifically focuses on the specific issues related to work.

\section{Reasons of working from home}

Before the outbreak of the virus, the largest part of respondents (80.5\%) declared little or no experience of SM, and a lot had simply never worked at home before the lockdown. Only a limited share (13\%) had already experienced SM for 4 days or more a week. Therefore, we have a sample of people that found themselves in a totally new working condition which occurred in an unexpected and sudden way. This should be considered carefully when discussing the findings of the analysis. What is notable is, however and rather unexpectedly, that the responses of the people with a previous SM experience are not dissimilar, on the whole, to non-experienced respondents.

As regards the reasons for SM, $85 \%$ of the sample indicated that they were working at home since they were ordered to do so, while $25 \%$ affirmed that they chose to do it (Figure 1). This confirms that the sample is made up by people with no previous experience of working from home or that they were not particularly eager to do so before the spread of the virus.

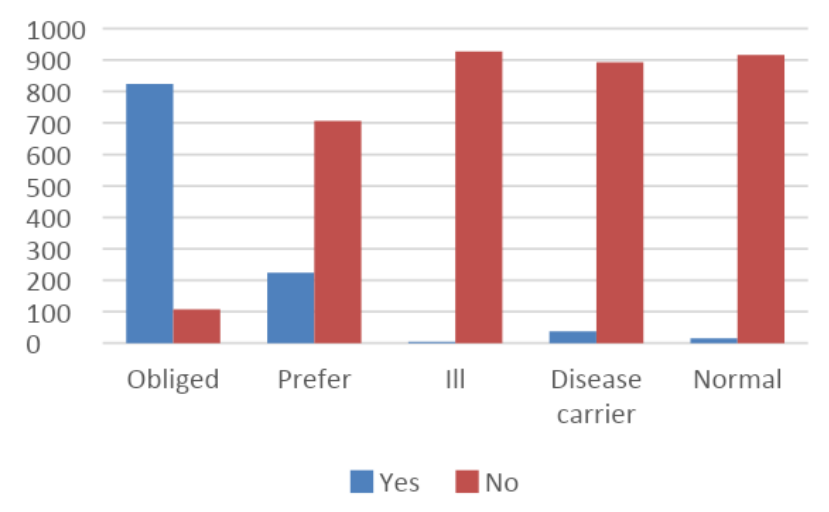

Figure 1. Reasons of working from home

Source: Authors' own research results

\section{Work situation and IT tools}

Participants were then asked to briefly outline their experience in relation to five workrelated aspects as outlined in Figure 2. 


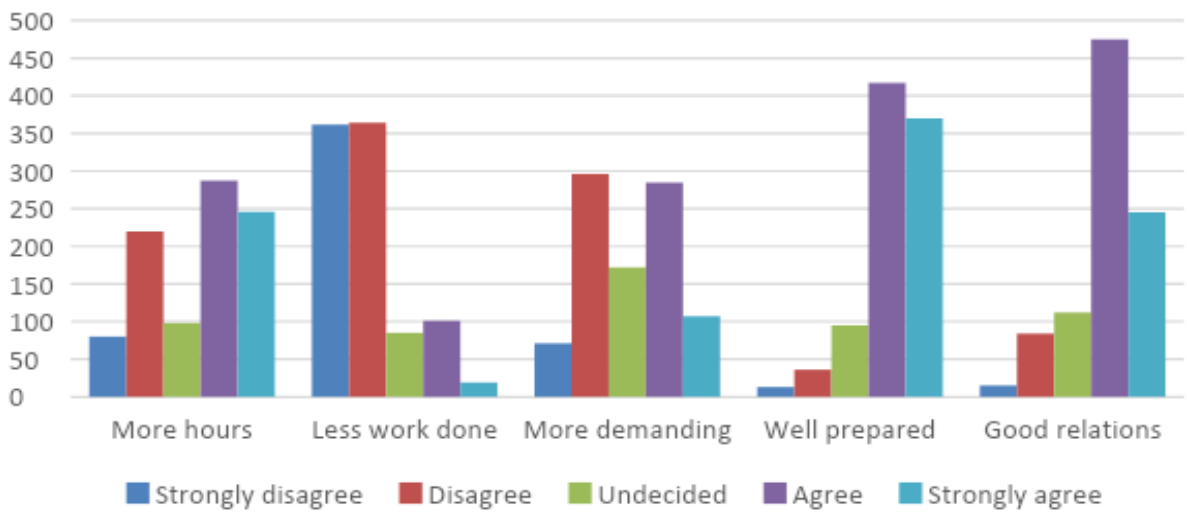

Figure 2. Work situation

Source: Authors' own research results

More than half of respondents affirmed that, with SM, they were working longer than normally, and only around $13 \%$ declared to have less work done. Probably, this is linked to the fact that their educational background or job experience were appropriate for facilitating their new working condition (as said, more than $67 \%$ of respondents have at least a bachelor's degree). Extremely interesting are responses to the question "is work in SM more demanding than normally?" that shows a polarized distribution between the two extreme answers of "more demanding" and "less demanding", although the average value of responses lays in the middle (3.07)

It is worth noting that most of the sample (77\%) stated that they were able to keep good relationships with colleagues even when working from home. As will be shown below, this must be ascribed due to an intense use of various digital communication systems.

We conducted a simple cluster analysis using as base variables the items concerning the working situation, whose result are shown in Table 2, where the average value of each segmentation variable, for the overall sample and for each segment, is indicated. Segmentation variables that are statistically different from the rest of the population are highlighted in red (lower) or green (higher). These variables distinguish each cluster from the others and identify its peculiar characteristics. Given the preliminary nature of the study, we applied the K-means clustering methods to the raw data without making any preliminary statistical elaborations (as e.g. a confirmatory factor analysis - Kaufman and Rousseeuw, 2009). Nevertheless, the results of the present and the following cluster analyses are interesting and deserve proper discussion.

Table 2. Results of the cluster analysis concerning the work situation

\begin{tabular}{|l|l|l|l|l|l|}
\hline & Population & Cluster 1 & Cluster 2 & Cluster 3 & Cluster 4 \\
\hline More hours & 3.43 & 1.86 & 4.08 & 2.16 & 4.51 \\
\hline Less work done & 1.98 & 1.56 & 1.57 & 3.72 & 2.00 \\
\hline More demanding & 3.07 & 2.59 & 2.15 & 2.79 & 4.19 \\
\hline Well prepared & 4.18 & 4.25 & 4.40 & 3.63 & 4.14 \\
\hline Good relations & 3.91 & 4.09 & 4.29 & 2.95 & 3.83 \\
\hline$\%$ & $100 \%$ & $26 \%$ & $27 \%$ & $12 \%$ & $35 \%$ \\
\hline
\end{tabular}

Source: Authors' own research results 
The cluster analysis identified 4 clusters that delineate realistic and plausible situations. This is a generally considered a sign of validity of the analysis (Sing, 2006). The clusters are:

- $\quad$ Cluster 1: people who have experienced no special difficulty, were able to carry out their normal job, and to keep good relations with colleagues. This cluster comprises $26 \%$ of respondents

- $\quad$ Cluster 2: very well-prepared people who were able perform their usual work, but by working more hours than usual. It is $27 \%$ of respondents

- $\quad$ Cluster 3: people who were less prepared, worked less than usual but getting less work done and being less able to keep good relations with colleagues. This is the smallest one ( $12 \%$ of the total sample)

- $\quad$ Cluster 4: people who perceived their work extremely demanding since they worked more hours to get the same results as usual and were less able to keep good relations with colleagues. This is the biggest one in terms of number of respondents (35\%).

We also made a discriminant analysis based on the demographic items included in the questionnaire, i.e. age, gender, education level, and number of children below 15 at home. This analysis did not produce any statistically significant results (i.e. clusters cannot be distinguished using these items), and hence these results are not reported here. Some implications are however mentioned in the next section.

The next question was about the employment of electronic communication systems supporting SM from home (Figure 3). Email, as expected, was the most commonly and intensively used system (96\% were using it always or often). Videoconference and shared online documents were very often used, other tools less frequently. Phone calls have been partially substituted by videoconference systems, very probably because these are now largely accessible, easy to use, and much richer media. Surprisingly, communication apps (as e.g. Messengers, and WhatsApp), that are very popular in private life, were little used in work context. Facebook and SMS are also inappropriate or outdated communication/interaction tools. Finally, other groupware programs are used by a small proportion of respondents. These are integrated tools that include various and sometimes special functions (email, web conferencing, chat online, videosharing, etc.) The overall picture shows that respondents resorted to a set of different systems to preserve their working relations. 


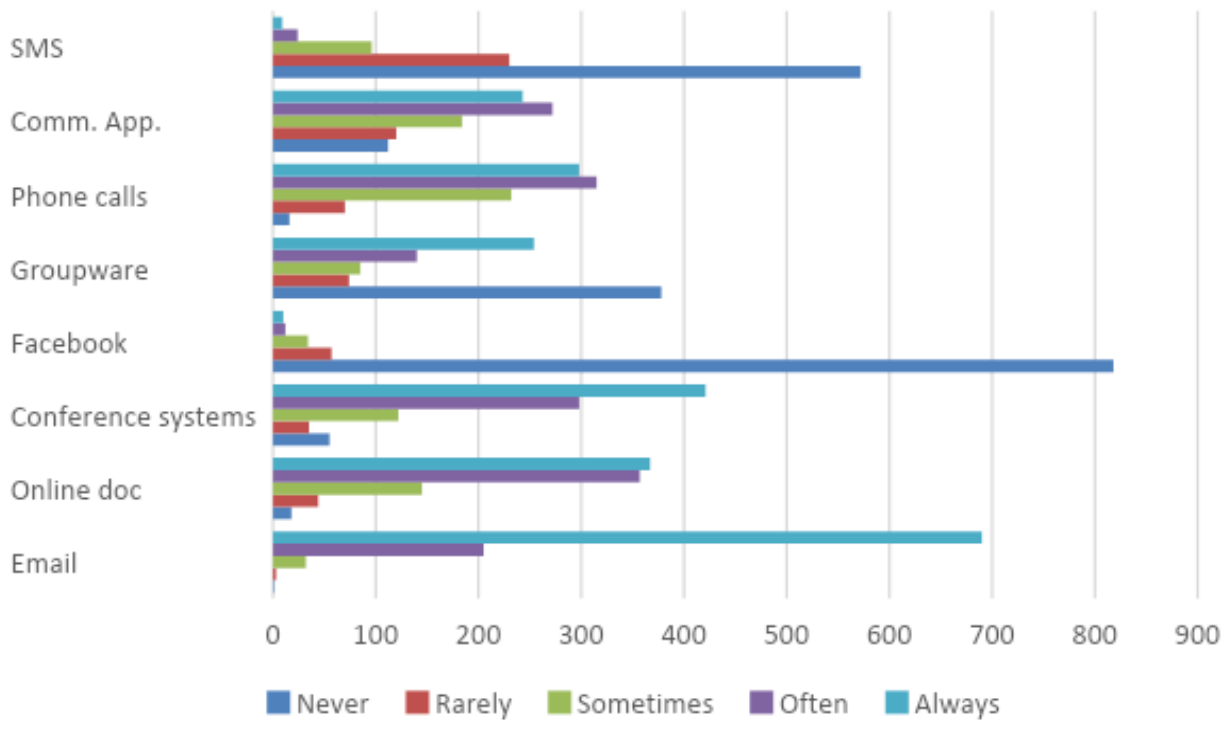

Figure 3. Use of communication systems

Source: Authors' own research results

We performed a cluster analysis on these data, to verify if it was possible to single out specific groups regarding the use of electronic communication systems. The analysis identified 4 clusters, that have a quite similar size, as follows (Table 3):

- $\quad$ Cluster 1: very frequent users of substantially all the systems

- $\quad$ Cluster 2: very frequent users of all the systems, apart from groupware programs. It is the biggest cluster

- $\quad$ Cluster 3: the least frequent users of all systems. It is the smallest cluster

- $\quad$ Cluster 4: the most frequent use of groupware systems (but infrequent users of all the other systems)

Table 3. Results of the cluster analysis concerning the use of communication systems

\begin{tabular}{|l|l|l|l|l|l|}
\hline & Population & Cluster 1 & Cluster 2 & Cluster 3 & Cluster 4 \\
\hline Email & 4.70 & 4.83 & 4.83 & 4.46 & 4.61 \\
\hline Shared documents & 4.09 & 4.37 & 4.40 & 3.43 & 3.98 \\
\hline Conference systems & 4.07 & 4.47 & 4.28 & 3.74 & 3.67 \\
\hline Facebook groups & 1.22 & 1.33 & 1.32 & 1.12 & 1.05 \\
\hline Other groupware & 2.80 & 4.33 & 1.20 & 1.16 & 4.41 \\
\hline Phone calls & 3.87 & 4.33 & 4.14 & 3.54 & 3.33 \\
\hline Communication app. & 3.44 & 4.39 & 4.43 & 2.40 & 2.17 \\
\hline SMS & 1.57 & 1.90 & 1.70 & 1.45 & 1.15 \\
\hline$\%$ & $100 \%$ & $27 \%$ & $28 \%$ & $21 \%$ & $24 \%$ \\
\hline
\end{tabular}

Source: Authors' own research results

The result is quite interesting especially if we consider that groupware systems are web-based office platforms that integrate the functions of the other separate systems. So, it is likely that adopters of these tools (Cluster 4) tend to replace all the single communication systems with an integrated suite. 
Table 4 shows a discriminant analysis considering the following descriptors: age, gender, education level, and number of contacts. In this case, as expected, the number of contacts influences the intensity of use of communication systems. The four clusters are different from one another especially as regards the number of daily contacts. In particular, the average number of contacts of the least frequent users (Cluster 2 ) is around 8 , while that of the very frequent users (Cluster1) is around 14, almost the double. There is also some difference (but less marked) in average age. The youngest people (more present in Cluster 1 and Cluster 4) are more frequent users of groupware programs. This can be explained considering their familiarity with digital communications and social media platforms, but it is a point that would deserve further investigation. Finally, gender and education level are apparently not a discriminating factor.

Table 4. Results of the discriminant analysis concerning the use of communication systems

\begin{tabular}{|l|l|l|l|l|l|}
\hline & Population & Cluster 1 & Cluster 2 & Cluster 3 & Cluster 4 \\
\hline Age & 3.79 & 3.68 & 4.05 & 3.99 & 3.43 \\
\hline Gender & 1.61 & 1.68 & 1.51 & 1.55 & 1.71 \\
\hline Education level & 6.17 & 6.24 & 6.21 & 6.22 & 6.02 \\
\hline Number of contacts & 3.75 & 4.19 & 3.87 & 3.13 & 3.65 \\
\hline \multicolumn{7}{|r|}{ Source: Authors' own research. }
\end{tabular}

Figure 4 confirms that, thanks to the previously recalled intense use of the systems, many people were able to stay in contact with a discrete number of people (about $43 \%$ of the sampled people communicated with ten persons or more each day).

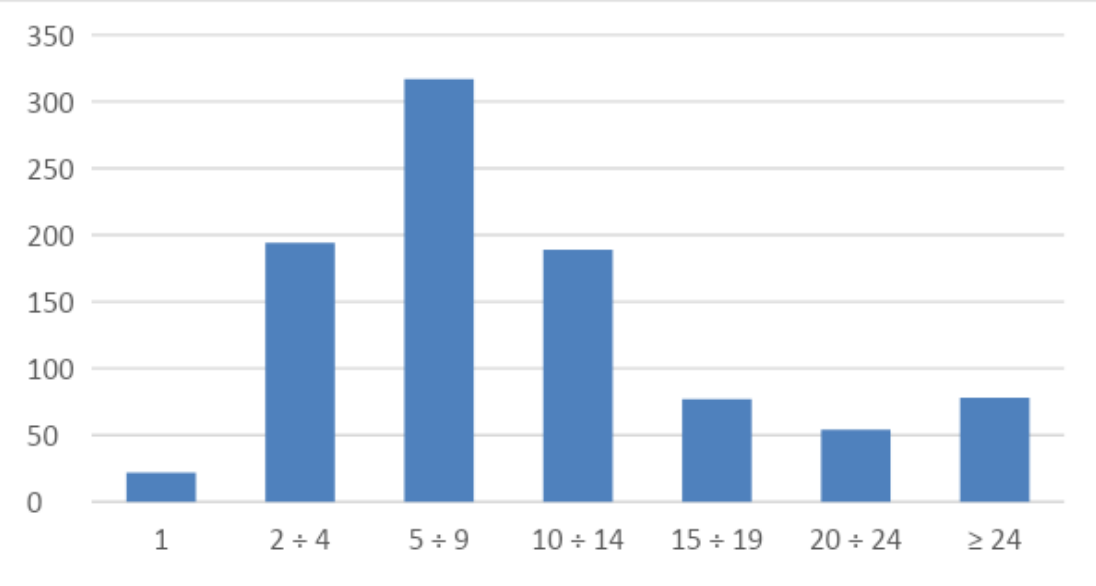

Figure 4. Number of people contacted daily

Source: Authors' own research results

\section{Advantages and disadvantages of SM}

Two key questions collected the feelings about factors that favored or hindered SM. As Figure 5 shows, respondents mostly appreciated that SM from home allowed them to "save transportation time" (93\% "strongly agreed" or "agreed"). Other highly appreciated aspects were the "possibility to eat and drink own food" (77\%), to better "focusing on work without interruptions" (69\%), and to be "close to family" (66\%). Conversely, "working in a more comfortable atmosphere" (35\%) is important but not for a great number of people, which 
partially contradicts what the existing literature often underlines. This is an interesting point to investigate more thoroughly in the future. Also, only for one third of the sample underlined "avoiding spending time in long meetings" (35\%): so, for these, online meetings are more productive that those in presence. Also, for few people SM resulted in "easier getting in contact with people" (19\%): consequently, while it appears that people kept in touch with colleagues frequently enough and were also able to keep good relations with colleagues (see table 4 and table 2) this was not perceived as a particularly decisive factor in favor of SM. Finally, no special advantage came from "having no-one looking over you" (only $12 \%$ ).

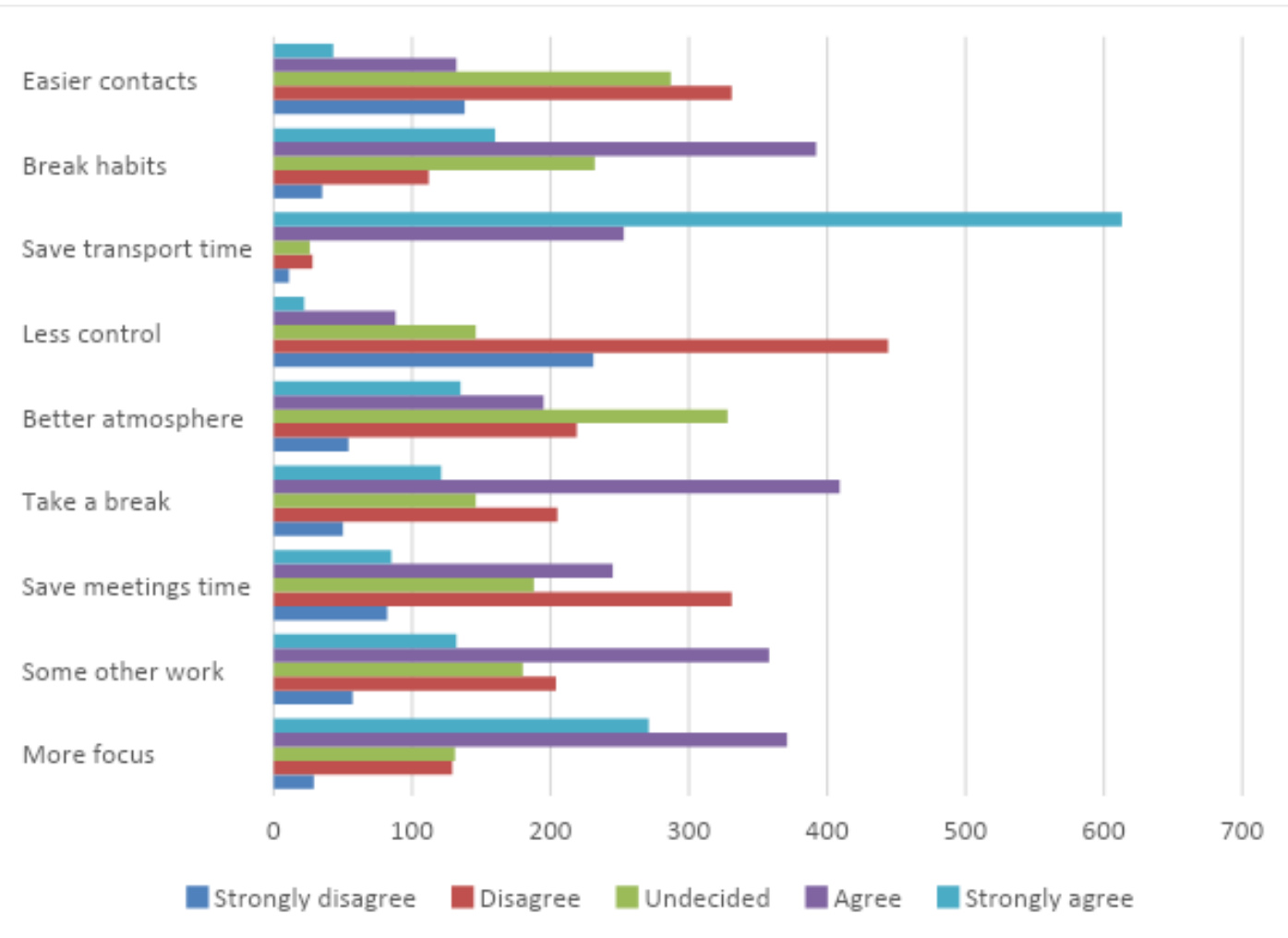

Figure 5. Factors benefiting working from home

Source: Authors' own research results

As regards the obstacles to SM and its negative factors, they were, on the whole, perceived (overall score: 2.25) as less significant than benefits (3.26). The most relevant negative factors are (Figure 6): feeling "tied with the computer to a greater extent than at workplace" (around 45\% of respondents "strongly agreed" or "agreed" with this statement); "difficult seeing colleagues or other people" (37\%), "missing physical equipment" (25\%) and concerns that "some tasks cannot be done from home" (25\%). A smaller share (between 14\% and 25\%) faced difficulties related to: "less interesting or not enough work", "missing access to needed data or documents", "bad physical conditions", and "missing physical equipment". Finally, some factors were considered as substantially irrelevant, such as: "not knowing what kind of work to do" (2\%) and "difficult to keep focused when alone" (5\%). 


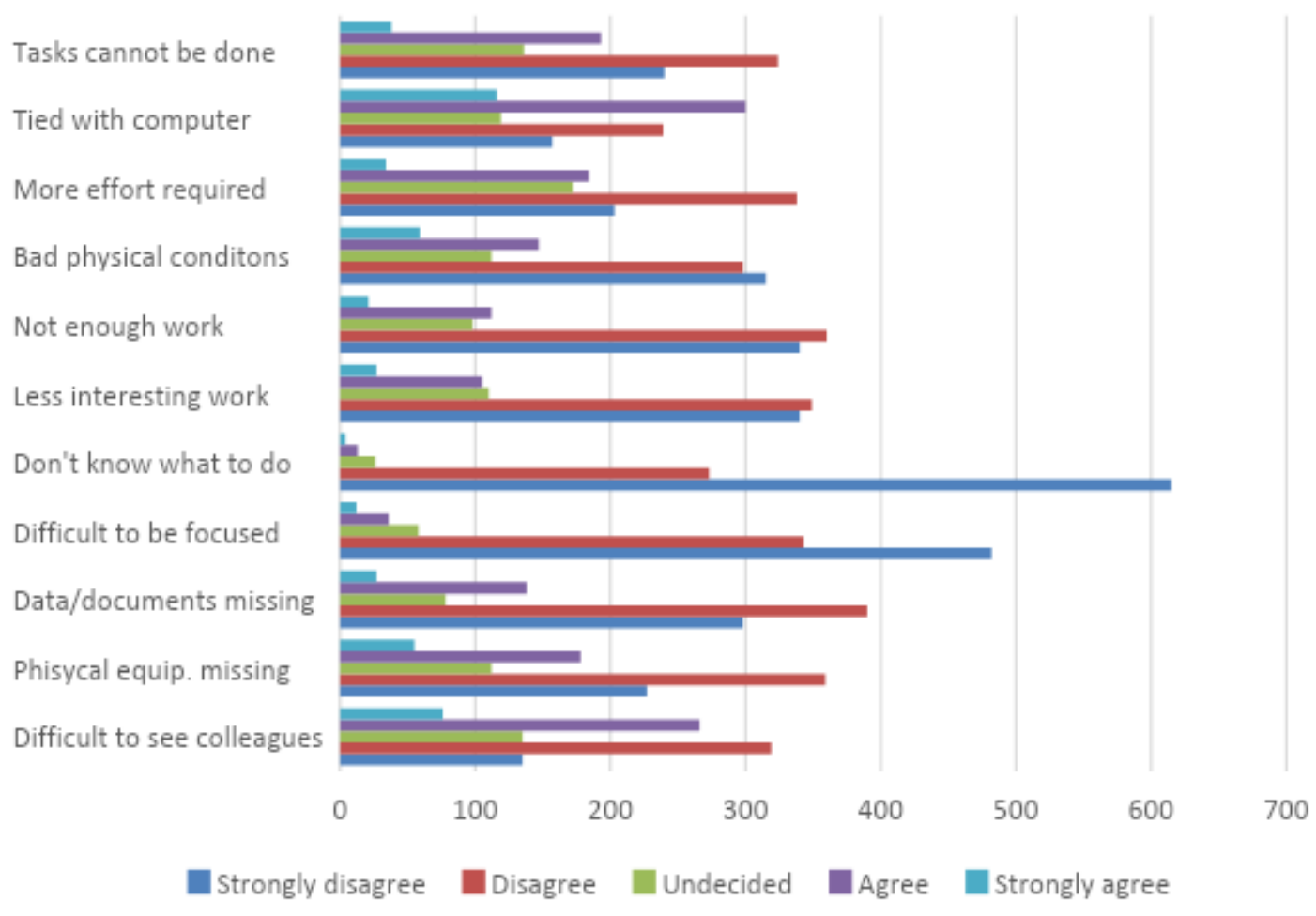

Figure 6. Factors hindering working from home

Source: Authors' own research results

We made a cluster analysis considering the whole set of positive and negative factors associated to SM. This made it possible to single out three clusters as follows (Table 5):

- $\quad$ Cluster 1: respondents who perceived lower benefits and higher difficulties of SM from home (29\% of respondents)

- $\quad$ Cluster 2: respondents who perceived low benefits but also low problems. It is the biggest cluster ( $45 \%$ of respondents)

- $\quad$ Cluster 3: respondents who perceives high benefits and low obstacles. It is the smallest cluster ( $26 \%$ of the sample).

These clusters are logically consistent. On the one hand, we have a group of people who are not satisfied at all with SM (Cluster 1), which is opposed by a group of very satisfied people (Cluster 3 ) of a similar size. In the middle, a consistent group of people did not experience special benefits nor problems, but apparently considered their house environment unsuitable for work. This is another signal that the opinion that working from home is "comfortable" for employees (as argued in some of the existing studies) is not necessarily supported.

A discriminant analysis based on the same demographic descriptors previously used was also performed. Again, no special difference between clusters was detected. 
Table 5. Results of the cluster analysis on factors that benefit and hinder working from home

\begin{tabular}{|l|l|l|l|l|}
\hline & Population & Cluster 1 & Cluster 2 & Custer 3 \\
\hline More focus & 3.78 & 3.23 & 3.72 & 4.49 \\
\hline Some other work & 3.33 & 3.11 & 3.14 & 3.88 \\
\hline Save meetings time & 2.91 & 2.58 & 2.79 & 3.50 \\
\hline Low control & 2.17 & 2.31 & 2.12 & 2.09 \\
\hline Save transport time & 4.53 & 4.30 & 4.52 & 4.81 \\
\hline Break habits & 3.57 & 3.33 & 3.46 & 4.02 \\
\hline Easier contacts & 2.58 & 2.14 & 2.49 & 3.23 \\
\hline Difficult to see colleagues & 2.82 & 3.67 & 2.79 & 1.91 \\
\hline Physical equip. missing & 2.44 & 3.51 & 2.22 & 1.60 \\
\hline Data/documents missing & 2.15 & 3.23 & 1.89 & 1.37 \\
\hline Difficult to be focused & 1.66 & 2.24 & 1.57 & 1.17 \\
\hline Don't know what to do & 1.41 & 1.75 & 1.37 & 1.09 \\
\hline Not interesting work & 2.07 & 3.06 & 1.87 & 1.30 \\
\hline Not sufficient tasks & 2.05 & 3.06 & 1.85 & 1.28 \\
\hline Bad physical conditions & 2.29 & 2.84 & 2.41 & 1.45 \\
\hline More effort required & 2.47 & 3.03 & 2.66 & 1.53 \\
\hline Tied with computer & 2.98 & 3.72 & 3.14 & 1.87 \\
\hline Tasks cannot be done & 2.43 & 3.66 & 2.22 & 1.41 \\
\hline \% & $100 \%$ & $29 \%$ & $45 \%$ & $26 \%$ \\
\hline
\end{tabular}

Source: Authors' own research results

\section{The overall situation}

The answers provided by respondents are in Figure 7 and confirm the overall picture that $33 \%$ of respondents consider the current situation more challenging than the normal one, $36 \%$ just as normal, and 31\% less challenging than normal.

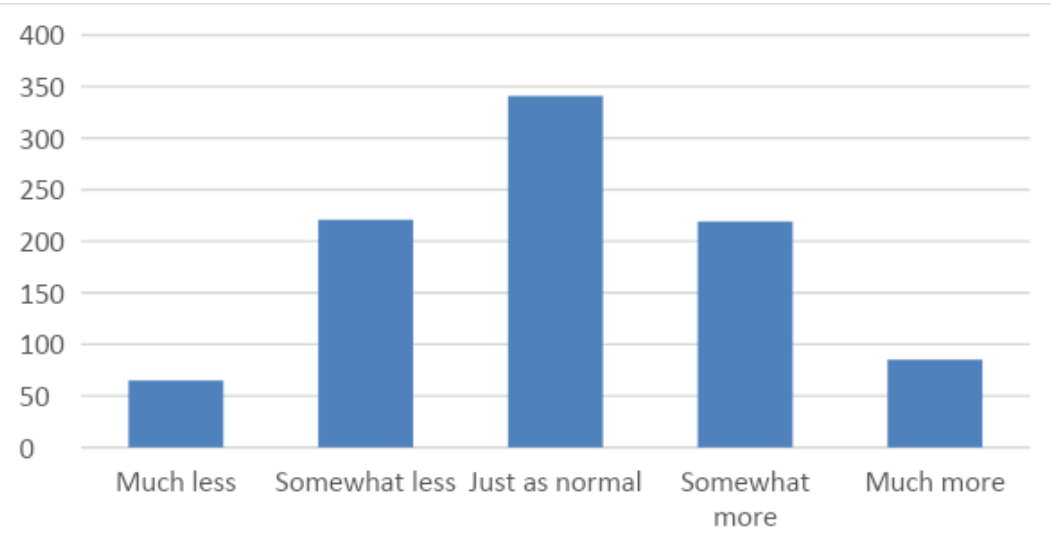

Figure 7. How much current work and life situation is challenging in comparison with the normal one Source: Authors' own research results 


\section{Discussion}

The analysis provides some stimulating insights. A particularly interesting is the overall view that respondents have about their SM experience. It doesn't emerge a clearly prevalent position but a rather balanced distribution among those who expressed a "positive" and those who expressed a "negative" evaluation of their experience, with a substantial group of people who considered the "new" situation neither better nor worse than the normal one. This is confirmed by the cluster analysis of benefiting and hindering factors that identified three groups of respondents whose statistical distribution is consistent with the previously mentioned picture. In short, it may be argued that the introduction of SM may not necessarily lead to a big "revolution" in working practices or lifestyles. This is a point to investigate further.

Another issue is that the usual "demographic" indicators, like those collected through the questionnaire (e.g. age, educational level, gender) do not allow to differentiate people and groups, especially between those who had positive experience from those who hadn't. This can be ascribed to the composition of the sample, or to the too simple statistical elaboration, but also to the fact that there may be more relevant descriptors that have not been included in the questionnaire (as e.g. sector and size of organization/company, or organizational role of respondents). It is a limitation of this study that can be considered in a future agenda.

Regarding more specific aspects, a crucial point is the efficiency/effectiveness of the "new" situation compared to the "old" working practices. Indeed, on the whole respondents signaled that SM makes it possible to perform the same work normally done and also to keep sufficiently good relationships with colleagues, but that they may be requested to work longer than usually, including those that were well prepared to SM. A marginal share (12\% of respondents) worked less than usual: probably this depends on the kind of job, that may be less suitable for SM - for example, employees who have a low level of autonomy and need to receive instructions continuously. Consequently, although SM is considered not so demanding (at least on average), it can be not necessarily efficient and effective, at least when it is not a free or agreed choice of employees and employers (like in the COVID situation). This may be confirmed by the fact that, to keep all the contacts needed to carry out their work, respondents had to use multiple electronic communication systems. Therefore, a not insignificant number of respondents felt strongly tied with (and probably stressed by) their computers and had to spend a lot of time in long online meetings.

As regards the employed communication system, email is still irreplaceable, but SM from home gave an incredible boost to the use of videoconference systems at the expenses of phone calls. Alternative communication apps (e.g. WhatsApp, Messenger) are, instead, less popular in SM. The case of groupware is special because the sample is divided into respondents that never used it (41\%) and those $(27 \%)$ for whom it is indispensable.

These results, together with the significant number of daily contacts needed to be maintained to make work done, confirms the relational essence of human work, and especially the intellectual - knowledge intensive - functions. This seems to be the factor that more strongly impacted on SM from home in the case of our sample: respondents who were less able to keep work relations with colleagues worked longer to get the usual results. As is well known, many companies and public organizations have often encouraged mutual sharing of knowledge between employees with various modalities, e.g.: open space offices, informal meetings, water dispensers, etc., based on the assumption that sharing knowledge 
can increase intellectual productivity of workers. Instead, SM apparently goes in the opposite directions and may make interactions and knowledge exchange more difficult and make some workers feel "isolated". In this regard, one may wonder if SM is really and always "smart".

Further comments can be made on the perceptions of problems and negative factors of SM. This can provide lessons to companies willing to implement or extend SM from home in the future. Among the negative aspects we can include: the uneasy contact with people, the impossibility to avoid long meetings, and working in an uncomfortable atmosphere. As previously said, the first two issues are linked with the relational nature of human work: it is not simple to find substitutes to face-to-face interactions. As regards the third aspect, this is related to the characteristics of the physical space where the work is done, characteristics that should be carefully evaluated before implementing the new form of SM from home. In other words, it cannot be expected that all employees can feel comfortable at their own homes, which can be small, not well connected, not organized for working, or simply a too distracting environment.

The most appreciated benefits ("saving transportation times", "eating and drinking own food" and "focusing on work without interruptions") are intrinsically connatural with SM from home and for this reason they don't require particular managerial interventions. "Having no control", instead, was not perceived as a benefit probably because our sample is mainly composed by high qualified knowledge workers who in general have a considerable autonomy. This also explain why the less perceived problem is "not knowing what to do". A point should be, however, made as regards the case of unskilled workers: for them, SM can be a challenge, and may require special measures.

Other negative aspects reflect what has been already mentioned, i.e. "feeling too much tied with computer" (a sort of technostress), "difficult to see colleagues" (a feeling of isolation), "missing physical equipment" and "thinking that some task cannot be done from home". First and second points confirm what was previous discussed, and the last two can be overcome by means of adequate planning and organization of SM. This was not possible in the current situation, that suddenly forced all employees to be locked down, but in the future appropriate planning can be possible.

\section{Conclusion}

This study illustrated a preliminary analysis of the results of an international survey aiming at collecting opinions about the experiences of SM from home in times of COVID-19. Particularly, it focused on data coming from respondents who, during the lockdown, stayed at home in Italy. Even though the data may be further processed, what has emerged so far contributes to increase our understanding about SM in general and from home especially and provides some food for thoughts for future research.

Compared to the existing pre-COVID literature, the study confirms some results of previous research and provides some additional insights. Apparently, it is confirmed that SM may be a positive (or, at least, not a so negative) solution for a large part of employees, especially knowledge workers that can perform their duties in substantial autonomy. However, this may imply longer and more stressful working hours, and a need to "be online" for a large part of the day. Generally speaking, we found a polarization of answers into two extremes - those that are substantially in favour and those that are not, which 
means that SM may not always be a positive solution for employees, at least if they need to adopt it suddenly and forcibly.

It is also confirmed that the kind of job - and the associated competences of employees - is a discriminating factor. Those that cannot work in autonomy, are not familiar with communication technologies, or are unable to keep fruitful relations with colleagues from remote, may be less productive in a SM condition. In addition, contrarily to what was generally assumed in the pre-COVID literature, working from home is not always a positive factor for employees: instead, it depends on the specific personal conditions.

As regards socialization and knowledge sharing, the survey shows that a large part of employees can be able to keep sufficiently good and fruitful interactions. However, this is also associated to the kind of job, and to the availability of appropriate communication technologies. Indeed, the study confirms that technology is a key factor and, in general, there is no "one-fits-all" solution. This implies that many employees must struggle to use different communication systems, which can increase their stress.

\section{Research implications}

The study provides some interesting indications about the positive and negative factors associated to SM from home, especially for highly qualified knowledge workers. In addition, findings show that some results of research on SM in pre-COVID times are confirmed, others are (or remain) debatable. The cluster analysis allowed to identify that there are groups of people who share similar opinions about a positive or a negative judgment of their SM from home experience, and on the advantages/disadvantages of this organizational solution. In addition, the demographic descriptors we included in the survey did not allow to discriminate between the different groups, which is also another interesting point. All the above paves the way for future research aimed, on the one hand, at confirming the results of this analysis when the emergency will be over and, on the other hand, at using different descriptive variables in order to understand if the different perceptions about working from can be linked to specific individual working conditions.

\section{Practical implications}

The study provides useful lessons regarding the potentially negative and positive effects (see the discussion section), that can be useful for managers that are willing to promote or extend SM in their companies. Firstly, the "smartness" of working from home cannot be (or should not be) taken for granted. In this regard, managers must be aware that working (only or prevalently) from home could diminish the intellectual productivity of their knowledge workers when it makes it difficult to exchange knowledge with colleagues. Secondly, a continuous online connection can stress workers and reduce productivity and interpersonal relationships. Companies should be aware of these negative aspects and must be prepared to take appropriate measures to contrast them.

\section{Limitations}

The study presents some limitations which should be considered when interpreting the results. Firstly, a non-probabilistic sample of respondents was used, which limits the generalizability of results. Secondly, answers given by respondents were influenced by the particular conditions imposed by the lockdown: people were suddenly ordered to stay at home together with their family and were not able to prepare themselves and arrange the 
environment for the new condition. Finally, a comparison with different countries will also be possible, considering that the survey has been submitted to many other national environments.

\section{References}

Alizadeh, T. (2013). Planning implications of telework: a policy analysis of the Sydney metropolitan strategy. Australian Planner, 50(4), 304-315.

Bélanger, F., \& Allport, C. D. (2008). Collaborative technologies in knowledge telework: an exploratory study. Information Systems Journal, 18(1), 101-121.

Bratianu, C. \& Bejinaru, R. (2019). The theory of knowledge fields: a thermodynamics approach. Systems, 7(2), 1-12.

Bratianu, C. \& Bejinaru, C. (2020). Knowledge dynamics: a thermodynamics approach. Kybernetes, 49(1), 6-21.

Brynjolfsson, E., Horton, J. J., Ozimek, A., Rock, D., Sharma, G., \& TuYe, H. Y. (2020). COVID-19 and remote work: An early look at us data. Cambridge: National Bureau of Economic Research, report $\mathrm{N}^{\circ} \mathrm{w} 27344$.

Dalkir, K. (2008). Computer-mediated knowledge sharing. In: Bolisani E. (ed.), Building the Knowledge Society on the Internet. Sharing and Exchanging Knowledge in Networked Environments, pp. 89-109. Hershey: IGI Global.

Dockery, A.M., \& Bawa S. (2014). Is working from home good work or bad work? Evidence from Australian employees. Australian Journal of Labour Economics, 17(2), 163.

Everitt, B. S., Landau, S., Leese, M., \& Stahl, D. (2011). Cluster Analysis. Chichester, UK: John Wiley \& Sons, $5^{\text {th }}$ ed.

Fogarty, H., Scott, P., \& Williams, S. (2011). The half-empty office: dilemmas in managing locational flexibility. New technology, work and employment, 26(3), 183-195.

Fragouli, E., \& Ilia, I. (2019). Working smart and not hard": key to maximize Employee efficiency? International Journal of Information, Business and Management, 11(2), 74111.

Gottlieb, C., Grobovšek, J., \& Poschke, M. (2020). Working from home across countries. COVID Economics, 1(8), 71-91.

Govindaraju, M., \& Sward, D. (2005). Effects of wireless mobile technology on employee work behavior and productivity: An Intel case study. Proceedings of IFIP TC8 WG 8.2 International Working Conference, Cleveland August 1-3, 349-351

Hassan, G.A.S., (2016). Smart work and efficiency at the workplace. Research Report, American University in Cairo, Cairo.

Ipsen, C., Kirchner, K., \& Hansen, J.P. (2020), Evidences of working from home in times of COVID-19. International survey conducted the first moths of the national lockdowns March-May 2020, Research Report, DTU, Technical University of Denmark, Lybgby, July.

Richardson, H., Kvasny, L., Jackson, P., Gharavi, H., \& Klobas, J. (2006). Technologies of the self: Virtual work and the inner panopticon. Information Technology \& People, July 1.

Kang, Y. S., \& Kwon, S. D. (2016). A Study on the Influencing Factors of Smart-Work Performance. Journal of Information Technology Applications and Management, 23(1), 61-77.

Kaufman, L., \& Rousseeuw, P. J. (2009). Finding groups in data: an introduction to cluster analysis. Hoboken, NJ: John Wiley \& Sons. 
Kim, Y. Y., \& Oh, S. (2015). What makes smart work successful? Overcoming the constraints of time geography. Proceedings of the 48th Hawaii International Conference on System Sciences (pp. 1038-1047).

Kim, Y. Y., Oh, S., Oh, H. M., \& Lee, J. E. (2017). Factors on Successful Adoption of Smart Work: A Case Study through Time Geography and Communication Cube Perspectives. International Information Institute (Tokyo). Information, 20(2A), 699712.

Langè, V., \& Gastaldi, L. (2020). Coping Italian Emergency COVID-19 through Smart Working: From Necessity to Opportunity. Journal of Mediterranean Knowledge, 5(1), 163-171.

Malik, A., Rosenberger, Philip J, Fitzgerald, M., \& Houlcroft, L. (2016). Factors affecting smart working: evidence from Australia. International Journal of Manpower, 37(6), 10421066.

Mallia, K. L., \& Ferris, S. P. (2000). Telework: A consideration of its impact on individuals and organizations. Electronic Journal of Communication, 10(3/4).

Nonaka, I. (1994). A dynamic theory of organizational knowledge creation. Organization Science, 5(1), 14-37.

Park, K. (2018). An Exploratory Study on Influence Factors for Expectation Effect of Smart Work and the Attitude Difference between Positions and Job Types. Journal of Information Technology Applications and Management, 25(4), 23-39.

Park, K., \& Kim, Y. J. (2013). Difference in Acceptance Level of Smart Work among Tendencies of Personal Leadership Styles. Journal of Digital Convergence, 11(11), 197-207.

Sarti, D., \& Torre, T. (2017). Is Smart Working a Win-Win Solution? First Evidence from the Field. In Addabbo, T., Ales, E., Curzi, Y., \& Senatori, I. (Eds.) Well-being at and Through Work. Torino: Giappichelli Editore, 231-251.

Sing, K. (2007). Quantitative social research methods, Thousand Oaks, Ca.: Sage.

Troup, C., \& Rose, J. (2012). Working from home: Do formal or informal telework arrangements provide better work-family outcomes? Community, Work \& Family, 15(4), 471-486.

van der Lippe, T., \& Lippényi, Z. (2020). Co-workers working from home and individual and team performance. New Technology, Work and Employment, 35(1), 60-79. 